\title{
MULTIDIMENSIONAL PHASE UNWRAPPING FOR CONSISTENT APF ESTIMATION
}

\author{
Roy A. Sivley and Joseph P. Havlicek \\ School of Electrical and Computer Engineering \\ University of Oklahoma, Norman, OK 73019 USA
}

\begin{abstract}
The instantaneous amplitude, phase, and frequency (APF) characterize a nonstationary signal on a fundamental level. In this paper, we seek to obtain a consistent multidimensional APF where the estimated phase and frequency agree and where the frequency can be obtained as the gradient of an analytical phase model. Currently existing multidimensional phase unwrapping algorithms are incapable of providing this because they erroneously assume bandlimits on the trajectory of the instantaneous frequency and thus suffer from the "phase aliasing" phenomenon. We present a new multidimensional phase unwrapping algorithm based on tensor product splines that does not make this assumption and thereby largely eliminates the deleterious effects of phase aliasing.
\end{abstract}

\section{INTRODUCTION}

The instantaneous amplitude, phase, and frequency (APF) characterize the nonstationary structure of a signal on a fundamental level. For a complex-valued signal $t: \mathbb{R}^{n} \rightarrow \mathbb{C}$ given by $t(\mathbf{x})=a(\mathbf{x}) \exp [j \varphi(\mathbf{x})]$, the APF $a(\mathbf{x}), \varphi(\mathbf{x})$, and $\nabla \varphi(\mathbf{x})$ are uniquely determined up to the branch of the phase (where it is assumed that $a(\mathbf{x})$ is positive semidefinite). For a real signal $s(\mathbf{x})=a(\mathbf{x}) \cos [\varphi(\mathbf{x})]$, they are ambiguous and additional constraints must be imposed to regularize the APF estimation problem. Various techniques based on differential operators and the analytic signal [1-3], quadratic demodulators including the Teager-Kaiser operator $[4,5]$, and other approaches have been used to estimate the instantaneous amplitude and instantaneous frequency (IF) in one and multiple dimensions. In most practical scenarios these algorithms are applied to a locally narrowband signal obtained by filtering or to discrete samples of such a signal. Since exact general amplitude and frequency algorithms are not known for these cases, approximations must be applied and the empirical IF field $\nabla \varphi(\mathbf{x})$ is generally nonconservative and characterized by estimation errors. This is of little concern in many analysis applications where

This work was supported in part by the U.S. Army Research Laboratory and the U.S. Army Research Office under grant W911NF-04-1-0221. such frequency estimates have been used with great success. However, the approximation errors are almost always problematic in synthesis applications where one seeks to reconstruct the signal $t(\mathbf{x})$ or $s(\mathbf{x})$ from the estimated amplitude and frequency. AM-FM image coding and modulation domain signal processing are examples of such applications.

When perfect reconstruction is the goal, it is desirable to estimate a consistent APF in the sense that the phase $\varphi$ and frequency $\nabla \varphi$ agree almost everywhere, or, in the discrete case, at least agree on the sampling lattice. A discrete approach that we are currently investigating for improving the frequency estimation accuracy involves fitting tensor product splines to the estimated phase. The splines provide a piecewise polynomial phase model that can be differentiated analytically to obtain a consistent APF. The principal branch of the phase can always be obtained by applying $\arctan (\cdot)$ to the real and imaginary components of a complex signal or by applying $\arccos (\cdot)$ to a real signal subsequent to dividing out the estimated amplitude. While this approach provides both a consistent APF and perfect reconstruction, the obtained frequency field $\nabla \varphi$ is characterized by undesirable high frequencies that do not correspond to any visually meaningful structure in the signal, but rather arise solely from branch cuts in the wrapped phase estimate. This fact limits the usefulness of the approach in analysis applications. Thus there is a critical need for a high-quality, multidimensional phase unwrapping algorithm so that the unwrapped phase can be fit with splines to obtain a consistent, perfect reconstruction APF involving visually meaningful frequencies that correspond to the perceptually significant structure of the signal.

However, multidimensional phase unwrapping is a difficult problem that has been studied extensively as it relates to a variety of applications where phase information plays an important role, including SAR interferometry [6] and magnetic resonance imaging [7]. In this paper we introduce a new multidimensional phase unwrapping algorithm based on tensor product splines. Although the support of the discrete Fourier spectrum of any signal is limited to $(-\pi, \pi]$ $\mathrm{rad} / \mathrm{sample}$, this new algorithm achieves an advantage by realizing that no such limit is in fact applicable to the IF. As a result, the "phase aliasing" problem typical of existing 
unwrapping techniques is largely eliminated.

\section{BACKGROUND}

Empirical phase signals obtained via arctan and arccos are available only as principal values (PV) that contain artificial discontinuities along the branch cuts. In a variety of applications, such phase signals are useful only after these artificial discontinuities have been removed or "unwrapped" by selectively adding integer multiples of $2 \pi$. Thus, the problem is to estimate the unwrapped phase

$$
\varphi(\mathbf{k})=\psi(\mathbf{k})+2 \pi b(\mathbf{k})
$$

from its principal values $\psi(\mathbf{k})$, where $\mathbf{k} \in \mathbb{Z}^{n}$ and $b: \mathbb{Z}^{n} \rightarrow$ $\mathbb{Z}$ indicates the branch to which each phase sample belongs.

Existing phase unwrapping methods have dealt with discrete signals by defining the IF as a finite difference (FD) [7-12]; however, Spagnolini [11] also notes that a differentiator could be approximated by an FIR filter or that the IF could be estimated by maximum likelihood techniques. The primary disadvantage to using any of these alternative methods is that they all restrict the IF to the range $(-\pi, \pi]$ $\mathrm{rad} /$ sample, which is a theoretical fallacy [13].

The two primary classes of existing phase unwrapping methods are path integration and least-squares (LS) estimation [8-12]. The former method relies on integration of the phase gradient field and, as such, is sensitive to noise and nonconservative field estimates. The latter method, however, has the ability to reduce the propagation of noise errors across the reconstructed phase image. Unfortunately, previous multidimensional LS phase unwrapping algorithms that define the IF as an FD assume that these differences never exceed $\pi$ in magnitude. When this assumption holds, the FD of the unwrapped phase $\varphi(\mathbf{k})$ is equal to the wrapped FD computed from the wrapped phase $\psi(\mathbf{k})$, i.e.,

$$
\nabla_{F D} \varphi(\mathbf{k})=W\left\{\nabla_{F D} \psi(\mathbf{k})\right\} .
$$

Here, $W\{\cdot\}$ is the wrapping operator that maps its argument to its modulo- $2 \pi$ equivalent value in $(-\pi, \pi]$. Thus, $W\left\{\nabla_{F D} \psi(\mathbf{k})\right\}$ can simply be accumulated to obtain the true phase $\varphi(\mathbf{k})$. Alternatively, when the assumption is violated, (2) holds only up to a multiple of $2 \pi$ [7], and the phase gradient field is nonconservative $[7,8,10]$. This condition is referred to as phase aliasing, and it can lead to gross errors in the estimation of the true phase.

\section{SPLINE-BASED PHASE UNWRAPPING}

To circumvent the notion of phase aliasing, we assume that the phase lies in the space of tensor product cubic splines, and using the method of Unser et al. [14-16], the spline processing may be efficiently implemented by means of digital filtering. This assumption allows the phase gradient field to be computed analytically and poses no constraints on the values assumed by the inter-pixel phase differences or the magnitude of the IF. Although other model-based unwrapping algorithms have been proposed (e.g. [17]), they are often too restrictive to apply to the entire phase signal and require block processing techniques.

We have investigated both path integration and LS phase unwrapping methods for this spline framework; however, only the LS solution is presented here. It is well-known that this LS solution is equivalent to the solution of Poisson's equation [12], and we impose the mirror-symmetry and periodic extension conditions also required by the filtering methods proposed in [12, 14-16].

Due to the nature of tensor product splines, the filtering is separable and, thus, only a 1-D treatment is presented here. Given the discrete phase signal $\varphi(k)$, the transfer function for the cubic spline phase derivative $u(k)$ is

$$
\frac{U(z)}{\Phi(z)}=\left(\frac{6}{z+4+z^{-1}}\right)\left(\frac{z-z^{-1}}{2}\right) .
$$

Define the phase average $\varphi_{d}(k)$ by the transfer function

$$
\frac{\Phi_{d}(z)}{\Phi(z)}=\frac{z-z^{-1}}{2}
$$

and the estimated phase average $u_{d}(k)$ by

$$
\frac{U_{d}(z)}{U(z)}=\frac{z+4+z^{-1}}{6} .
$$

Thus, we can filter the derivative estimate $u(k)$ to get an approximation for the phase average as defined in (4).

For a 2-D phase signal with respective horizontal and vertical gradients $u(j, k)$ and $v(j, k)$, the transfer functions (4) and (5) can be used to write the LS error function

$$
\begin{aligned}
\varepsilon_{L S}=\sum_{j, k}[ & \left.\varphi_{d u}(j, k)-u_{d}(j, k)\right]^{2} \\
& +\left[\varphi_{d v}(j, k)-v_{d}(j, k)\right]^{2} .
\end{aligned}
$$

Differentiating this error with respect to $\varphi(j, k)$ and setting the result equal to zero gives the system of equations (7), where (5) has been used to write the system in terms of the IF estimates $u(j, k)$ and $v(j, k)$. This system is similar in form to that derived in [12], and because the same mirror-symmetry and periodic extension were used, (7) can be solved using a cosine transform implemented by FFT's.

In 2-D, there are four FFT coefficients that are undetermined and must be estimated to make the LS solution unique and ensure that each unwrapped phase sample is on the correct branch. Subsequent to estimating three of the coefficients, the dc coefficient can be estimated in a manner similar to that of [7], where the constant is the average of the 


$$
\begin{aligned}
& (\varphi(j+2, k)-2 \varphi(j, k)+\varphi(j-2, k))+(\varphi(j, k+2)-2 \varphi(j, k)+\varphi(j, k-2))= \\
& \quad \frac{1}{3}[u(j+2, k)+4 u(j+1, k)-4 u(j-1, k)-u(j-2, k)+v(j, k+2)+4 v(j, k+1)-4 v(j, k-1)-v(j, k-2)]
\end{aligned}
$$

wrapped differences between the unwrapped and wrapped phase values

$$
\varphi_{d c}=\operatorname{avg}_{j, k \in D}\{W\{\psi(j, k)-\varphi(j, k)\}\} .
$$

However, we propose that this average only be computed over regions $D$ where the error in the LS solution is below an empirical threshold. Lastly, due to errors in the IF estimation, the LS solution may not be congruent (modulo- $2 \pi$ ) with the original phase values; the methods of [7] or [17] may be used to map each phase estimate to its nearest congruent value.

\section{EXPERIMENTAL RESULTS}

The algorithm proposed in Section 3 was tested using two synthetic images and one natural image. The spline derivative (3) was used to estimate the IF. The three original (wrapped) phase images are shown in Fig. 1(a)-(c). The proposed algorithm unwraps the phases from Fig. 1(a) and (b) perfectly, as shown in Fig. 1(d) and (e). However, this algorithm has more difficulty with the measured phase in Fig. 1(c): a few fringe lines can still be seen in the unwrapped phase of Fig. 1(f). Nevertheless, the IF calculated by taking the gradient of the unwrapped phase is consistent almost everywhere. It is interesting to note that some of the fringe lines (e.g., those in the upper left and right of the image) are in agreement with the IF estimates and do not indicate an error in the unwrapping. Alternatively, the fringe lines in the lower right of the image do not agree with the IF and result from poor IF estimates in that region. It should be noted that the LS solution will never contain fringe lines. However, fringe lines are sometimes introduced through estimation of the four undetermined FFT coefficients which disambiguate the Poisson equation solution and also as a result of forcing the unwrapped and wrapped phases to be congruent. Different estimates for the four undetermined coefficients can generally produce different fringe paths.

Existing algorithms refer to the fringe line endpoints as singular points and note that they result from nonconservative gradient field estimates caused by phase aliasing. Because our model precludes the existence of phase aliasing, there are only two possible explanations for these singular points: poor IF estimation and poor estimation of the undetermined transform coefficients. Specifically, we have observed that the singular points invariably correspond to samples where the APF becomes ambiguous due to a vanishingly small amplitude. Additionally, while the IF calculated from the unwrapped phase is in good agreement with previous estimates, the unwrapping algorithm proposed here can be improved by using IF estimates that explicitly account for ambiguous phase at samples where the amplitude is small.

\section{CONCLUSION}

We presented a new multidimensional phase unwrapping algorithm based on tensor product splines that, for the first time, has the potential to deliver a consistent APF estimate. The assumed spline models are sufficiently general to apply to entire signal domains and do not restrict the range of the inter-pixel phase differences or of the instantaneous frequency magnitude. This provides the fundamental framework for new, improved methods of studying phase information and has the potential to overcome the limitations inherent in currently existing phase unwrapping algorithms.

\section{REFERENCES}

[1] L. Cohen, Time-Frequency Analysis, Prentice Hall, Englewood Cliffs, NJ, 1995.

[2] D. Vakman, "On the analytic signal, the Teager-Kaiser energy algorithm, and other methods for defining amplitude and frequency," IEEE Trans. Signal Proc., vol. 44, no. 4, pp. 791-797, April 1996.

[3] J.P. Havlicek, D.S. Harding, and A.C. Bovik, "Multidimensional quasi-eigenfunction approximations and multicomponent AM-FM models," IEEE Trans. Image Proc., vol. 9, no. 2, pp. 227-242, Feb. 2000.

[4] P. Maragos, J. F. Kaiser, and T. F. Quatieri, "Energy separation in signal modulations with applications to speech analysis," IEEE Trans. Signal Proc., vol. 41, no. 10, pp. 3024-3051, October 1993.

[5] P. Maragos and A. C. Bovik, "Image demodulation using multidimensional energy separation," J. Opt. Soc. Amer. A, vol. 12, no. 9, pp. 1867-1876, September 1995.

[6] R. Bamler, N. Adam, G.W. Davidson, and D. Just, "Noise-induced slope distortion in 2-D phase unwrapping by linear estimators with application to SAR interferometry," IEEE Trans. Geosci. Remote Sens., vol. 36, no. 3, pp. 913-921, May 1998. 


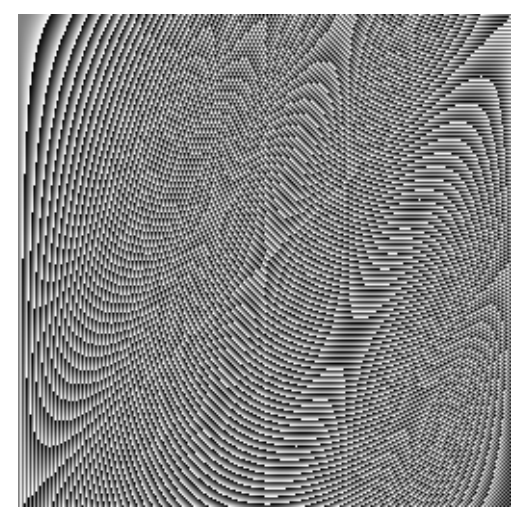

(a)

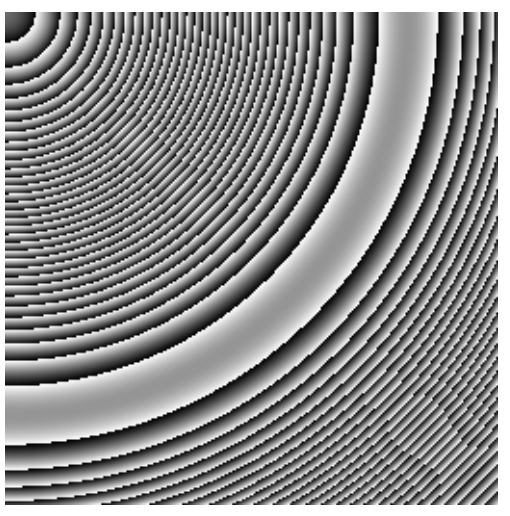

(b)

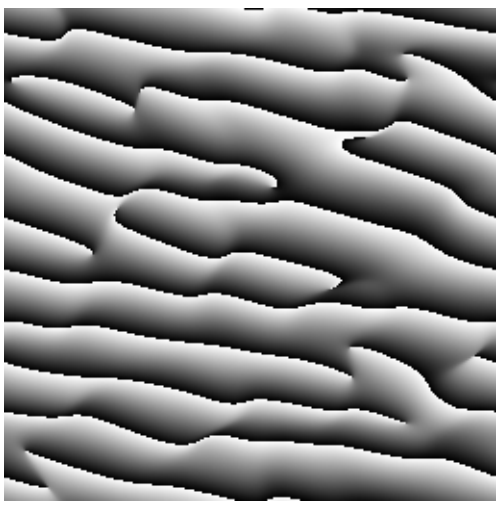

(c)

(f)

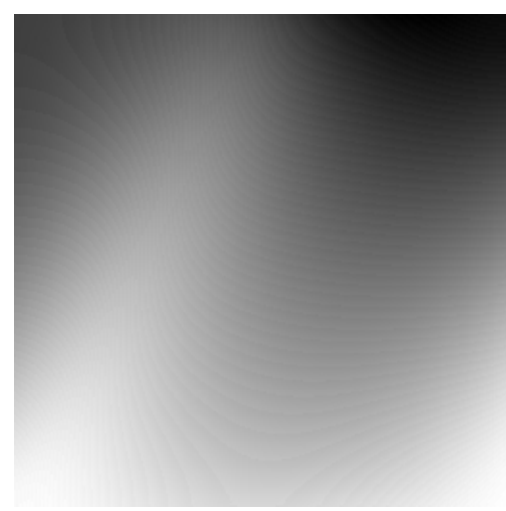

(d)

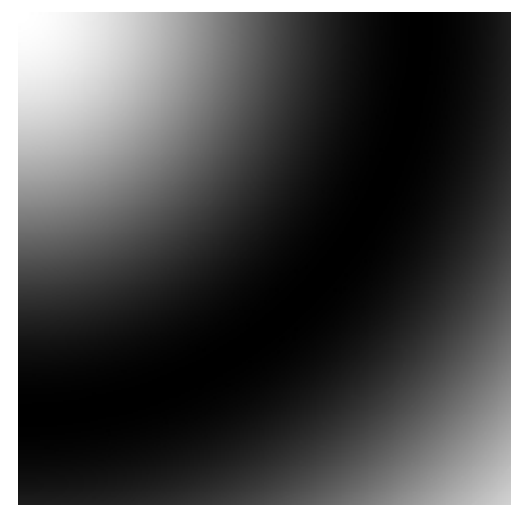

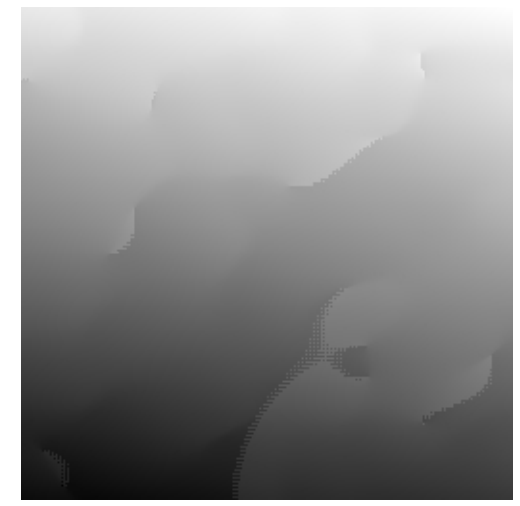

(e)

Fig. 1. Phase unwrapping results for synthetic and measured (natural) phase images. (a), (b) Synthetic wrapped phase images. (c) Wrapped phase image measured from the Gabor filterbank of [3] in response to a natural Brodatz texture. (d), (e) Unwrapped phases from (a) and (b), respectively. (f) Unwrapped phase from (c).

[7] S.M.-H. Song, S. Napel, N.J. Pelc, and G.H. Glover, "Phase unwrapping of MR phase images using Poisson equation," IEEE Trans. Image Proc., vol. 4, no. 5, pp. 667-676, May 1995.

[8] H. Takajo and T. Takahashi, "Least-squares phase estimation from the phase difference," J. Opt. Soc. Am. $A$, vol. 5, no. 3, pp. 416-425, Mar. 1988.

[9] H. Takajo and T. Takahashi, "Noniterative method for obtaining the exact solution for the normal equation in the least-squares phase estimation from the phase difference," J. Opt. Soc. Am. A, vol. 5, no. 11, pp. 1818-1827, Nov. 1988.

[10] U. Spagnolini, "2-D phase unwrapping and phase aliasing," Geophys., vol. 58, no. 9, pp. 1324-1334, Sep. 1993.

[11] U. Spagnolini, "2-D phase unwrapping and instantaneous frequency estimation," IEEE Trans. Geosci. Remote Sens., vol. 33, no. 3, pp. 579-589, May 1995.
[12] M.D. Pritt and J.S. Shipman, "Least-squares twodimensional phase unwrapping using FFT's," IEEE Trans. Geosci. Remote Sens., vol. 32, no. 3, pp. 706708, May 1994.

[13] L. Mandel, "Interpretation of instantaneous frequencies,” Am. J. Phys., vol. 42, pp. 840-846, 1974.

[14] M. Unser, "Splines: A perfect fit for signal and image processing," IEEE Signal Proc. Mag., vol. 16, no. 6, pp. 22-38, Nov. 1999.

[15] M. Unser, A. Aldroubi, and M. Eden, "B-spline signal processing: Part I-theory," IEEE Trans. Signal Proc., vol. 41, no. 2, pp. 821-833, Feb. 1993.

[16] M. Unser, A. Aldroubi, and M. Eden, "B-spline signal processing: Part II-efficient design and applications," IEEE Trans. Signal Proc., vol. 41, no. 2, pp. 834-848, Feb. 1993.

[17] B. Friedlander and J.M. Francos, "Model based phase unwrapping of 2-D signals," IEEE Trans. Signal Proc., vol. 44, no. 12, pp. 2999-3007, Dec. 1996. 